\title{
Masa septal interauricular: más allá de la hipertrofia lipomatosa
}

\author{
Álvaro Riesgo García* \\ Javier Ibero Valencia* \\ José Gavira Gómez*
}

\author{
Agnes Díaz Dorronsoro* \\ Gorka Bastarrika Alemañ ${ }^{* *}$
}

\section{Correspondencia}

Álvaro Riesgo García ariesgo@unav.es

* Departamento de Cardiología y Cirugía Cardíaca. Clínica Universidad de Navarra. España
* Servicio de Radiología. Clínica Universidad de Navarra. España

Recibido: $25 / 03 / 2020$

Aceptado: 12/06/2020

En línea: 31/07/2020

Citar como: Riesgo-García A, Ibero-Valencia J, Gavira-Gómez J, Díaz-Dorronsoro A, Bastarrika-Alemañ G. Masa septal interauricular: más allá de la hipertrofia lipomatosa. Rev Ecocar Pract (RETIC). 2020 (Jul); 3 (2): 42-44. doi: 10.37615/retic.v3n2a13.

Cite this as: Riesgo-García A, Ibero-Valencia J, Gavira-Gómez J, Díaz-Dorronsoro A, Bastarrika-Alemañ G. Interatrial septal mass: beyond lipomatous hypertrophy. Rev Ecocar Pract (RETIC). 2020 (Jul); 3 (2): 42-44. doi: 10.37615/retic.v3n2a13.

\section{Palabras clave \\ $\triangleright$ Mieloma múltiple \\ $\triangleright$ Plasmocitoma \\ $\triangleright$ Hiperplasia septal lipomatosa}

\section{Keywords \\ $\triangleright$ Multiple myeloma \\ $\triangleright$ Plasmacytoma \\ $\triangleright$ Lipomatous septal hyperplasia}

\section{Presentación del caso}

Varón de 56 años, exfumador, con antecedentes de hipertensión arterial, hiperuricemia y tromboembolismo pulmonar incidental, en tratamiento anticoagulante. Diagnosticado de mieloma múltiple tipo lgG lambda en mayo de 2009, recibió hasta seis líneas de tratamiento quimioterápico y trasplante autólogo de sangre periférica. En 2018, se constata analíticamente progresión de su enfermedad, realizándose tomografía computarizada de baja dosis que objetiva múltiple infiltración de partes blandas, costillas y huesos largos, compatibles con plasmocitomas extramedulares. Es remitido al Servicio de Cardiología para valoración, encontrándose en clase funcional I, con electrocardiograma en ritmo sinusal y sin alteraciones electrocardiográficas a excepción de patrón S1Q3T3,ya conocido.

Se le realizó una ecocardiografía transtorácica (ETT) de control postratamiento con antraciclinas (Figura 1), documentándose engrosamiento llamativo de septo interauricular. No se observaron otras alteraciones.

Dados los hallazgos ecocardiográficos se decide realizar una resonancia cardíaca (Figura 2). Se observó una masa lobulada que infiltraba el septo interauricular y que se extendía a la pared inferior de la aurícula izquierda (Vídeo 1). La masa presentaba una intensidad de señal heterogénea respecto al miocardio. Era ligeramente hiperintensa en secuencias T1. Presentaba aumento de la intensidad de señal en secuencias STIR-T2 y tiempos prolongados en los mapas paramétricos: T2 post de 71 ms (Figura 3 y Figura 4). En su crecimiento obstruía parcialmente la desembocadura de la vena cava inferior en aurícula derecha y contactaba ampliamente con el suelo de la aurícula izquierda (Vídeo 2), sin infiltración evidente de la misma. Medía unos $46 \times 22 \times 22 \mathrm{~mm}$. No mostraba anulación en las secuencias de supresión grasa y, durante la perfusión, presentaba una curva de captación de contraste de características malignas (Vídeo 3). La captación de contraste por parte de la masa en las secuencias de realce tardío era evidente y heterogénea (Figura 5). No se objetivó captación tardía de gadolinio sugestiva de enfermedad miocárdica, ni trombos intracavitarios en realce precoz. El volumen extracelular del miocardio calculado en los mapas T1 para un hematocrito del 35,2\% era del 29\%. En la lesión estudiada era del 54\%. Además, se observaron múltiples nódulos pulmonares bilaterales, el mayor de ellos en LII de 23 mm, en relación con su proceso neoplásico (Vídeo 4). Dado el contexto clínico y el comportamiento sugestivo de malignidad en la resonancia, se diagnosticó la masa de plasmocitoma cardíaco.

Asimismo, se le realizó una tomografía de emisión de positrones con 18-fluordeoxiglucosa (PET-18FDG) de estadificación oncológica (Figura 6) que documentó infiltración de septo interauricular (SUV máx $=11,5$ ), además de abundante infiltración plasmocítica de partes blandas, compatible con enfermedad extramedular extensa. 


\section{Estudio por imagen}

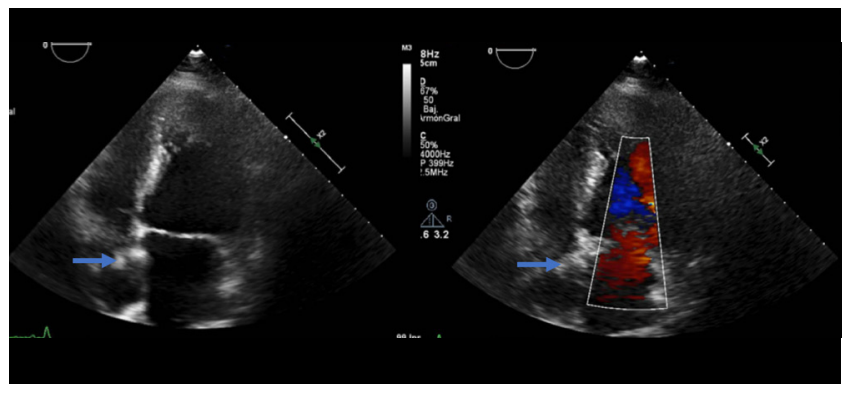

Figura 1. ETT, plano apical de cuatro camaras con y sin Doppler color. La flecha azul señala la zona de engrosamiento del septo interauricular

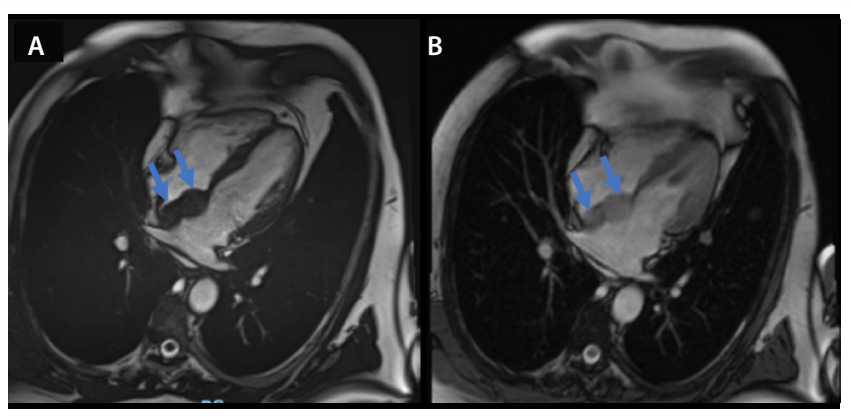

Figura 2. RM de corazón. A: secuencia de cine de cuatro cámaras; B: secuencia de realce precoz. Las flechas azules señalan la zona de engrosamiento del septo interauricular

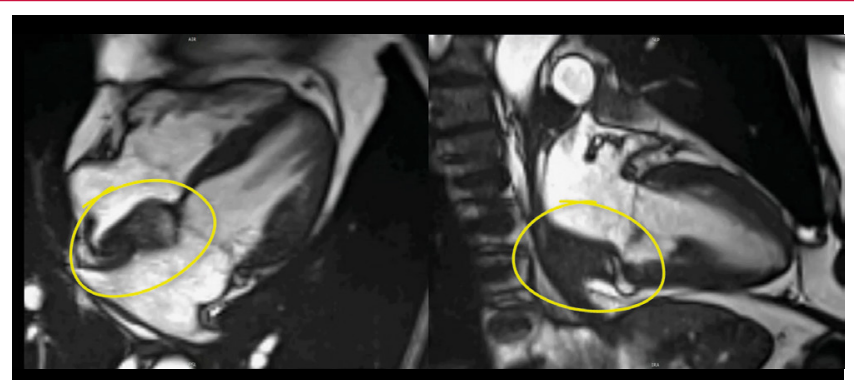

Vídeo 1. RM de corazón secuencias de cine en planos de cuatro y dos cámaras. Se observa una masa lobulada (círculo amarillo) que infiltra el septo interauricular y se extiende a la pared inferior de la aurícula izquierda

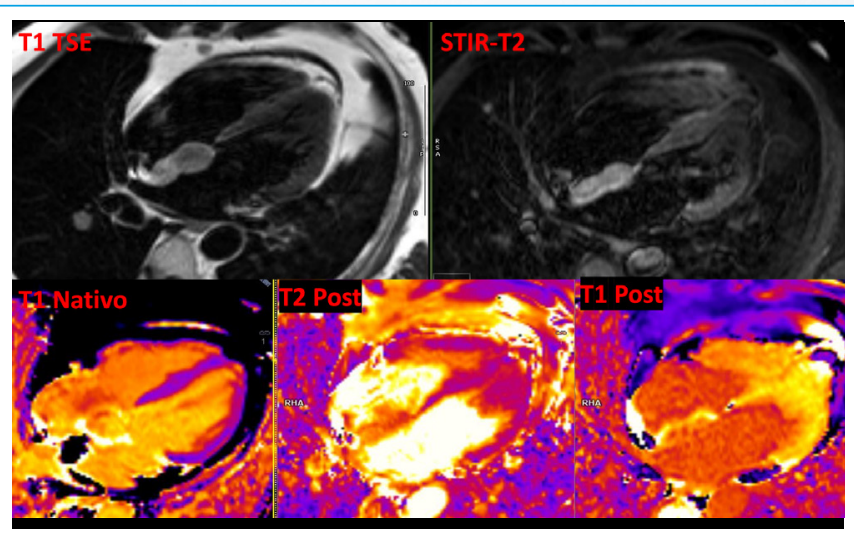

Figura 3. RM de corazón. Secuencias potenciada en T1 (arriba a la izquierda), secuencia T2-STIR (arriba a la derecha), secuencias paramétricas (abajo)

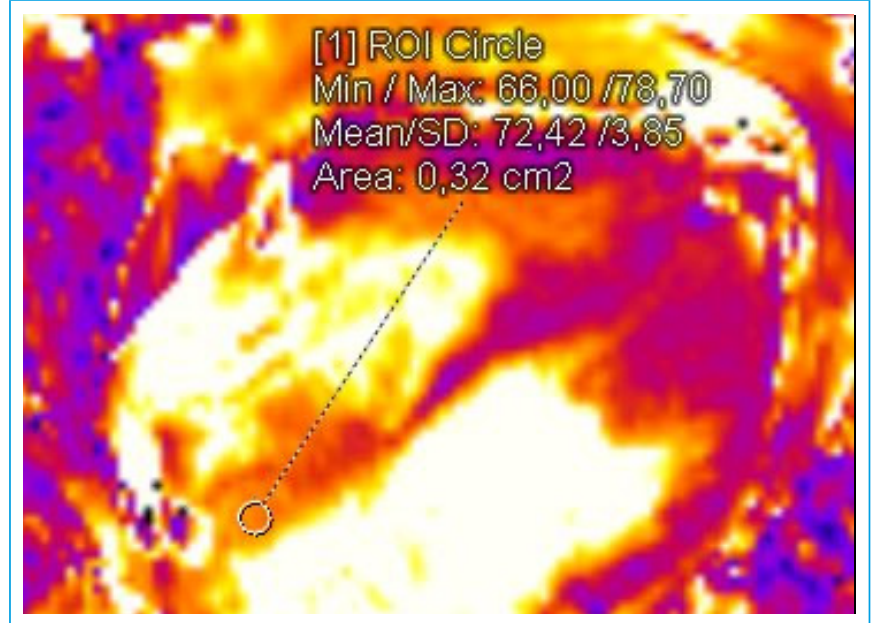

Figura 4. Secuencia paramétrica T2 post-contraste (72 ms)

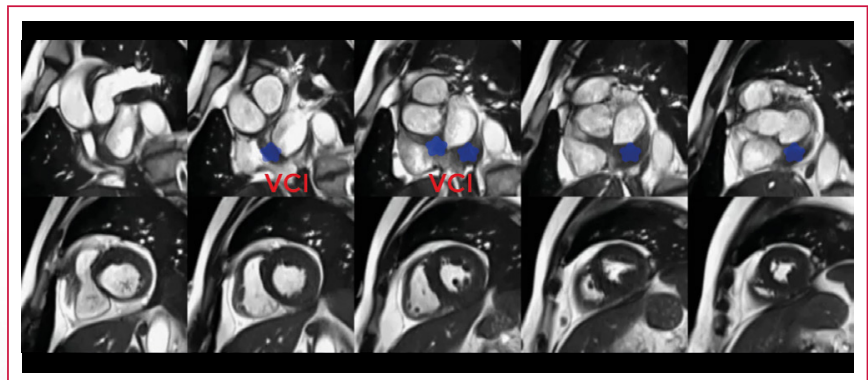

Vídeo 2. RM de corazón secuencia de cine eje corto. En azul se destaca la masa (VCl: vena cava inferior)

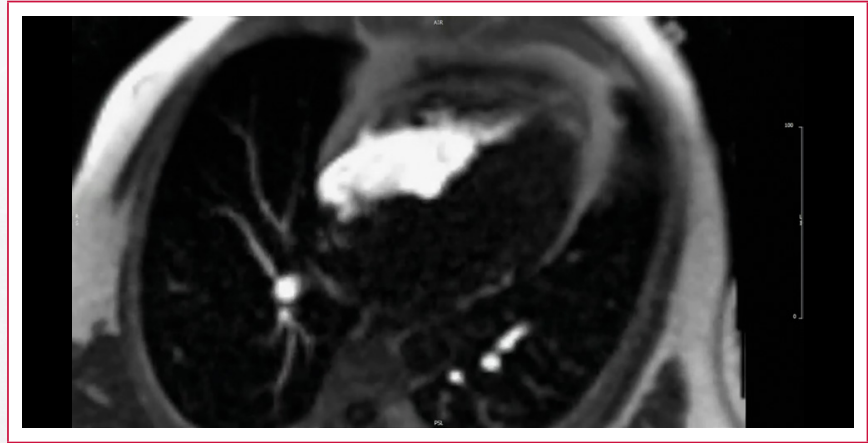

Vídeo 3. RM de corazón secuencia de perfusión. Se observa captación de contraste difusa y heterogénea por parte de la masa (indica que está vascularizada)

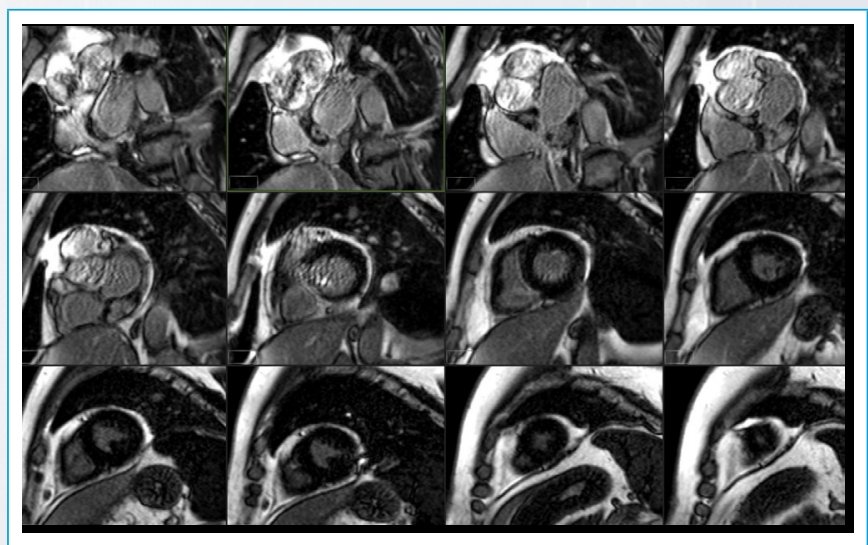

Figura 5. Secuencia de realce tardío en eje corto. Se observa una captación de contraste por parte de la masa evidente y heterogénea 


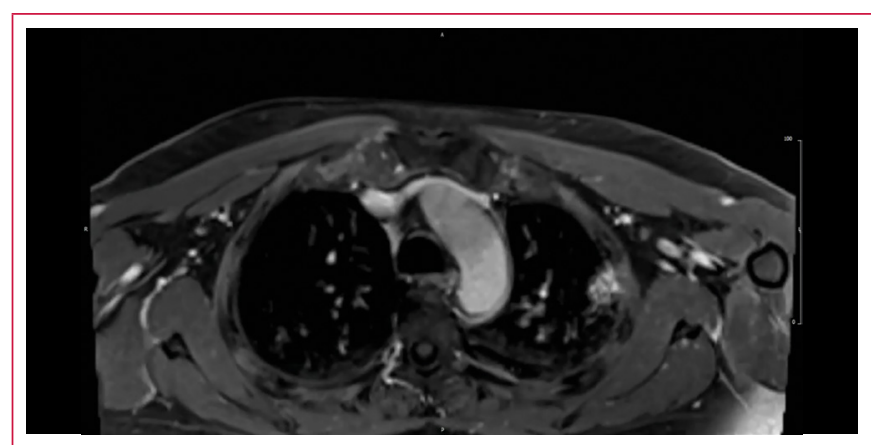

Vídeo 4. RM de corazón secuencia VIBE: anatomía torácica después de la administración de contraste. Afectación pulmonar de características malignas: múltiples nódulos pulmonares bilaterales, el mayor de ellos en LII de $23 \mathrm{~mm}$

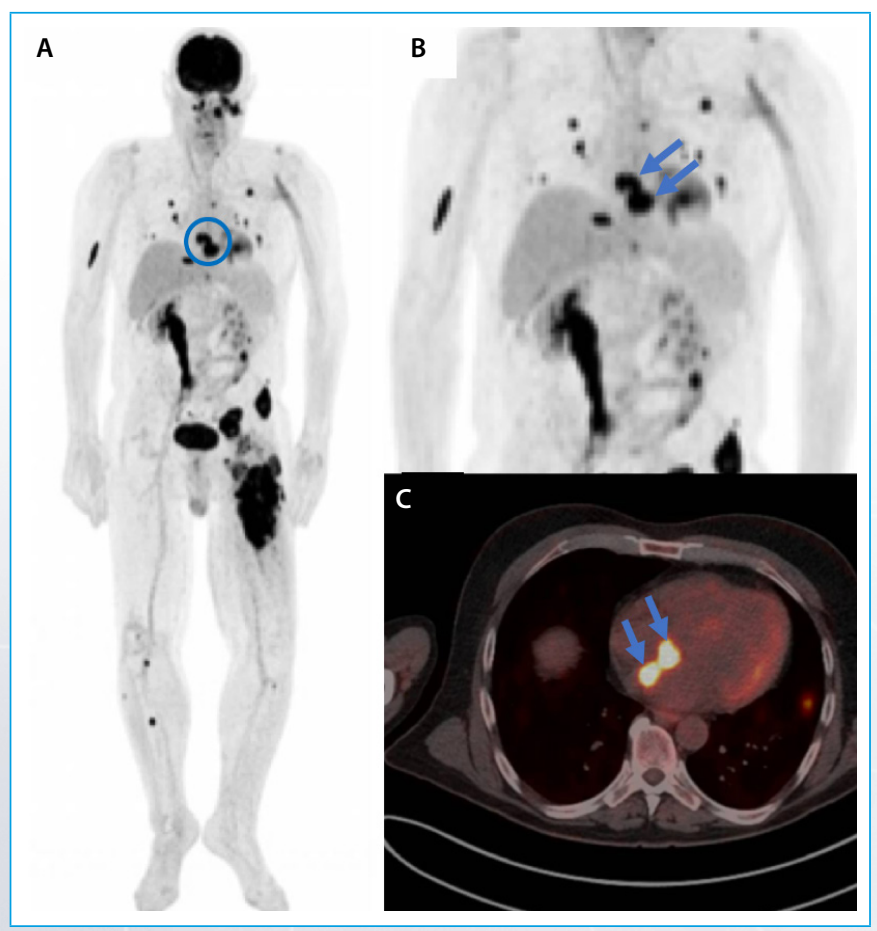

Figura 6. PET-TC. A: plano coronal infiltración de partes blandas, costillas y huesos largos, compatible con plasmocitomas extramedulares; B: detalle del plano coronal; C: imagen de fusión PET-TC, corte axial. El engrosamiento del septo interauricular se destaca mediante un círculo azul (A) y flechas azules (B y C)

\section{Discusión}

El mieloma múltiple se caracteriza por la proliferación de células plasmáticas en la médula ósea, resultando en una producción clonal de inmunoglobulinas. La enfermedad extramedular plasmocítica constituye el $4 \%$ del total de las neoplasias de células plasmáticas, siendo la afectación cardíaca extremadamente inusual(1). Su presentación como hallazgo incidental en pacientes asintomáticos también es infrecuente, siendo habitual que debuten con clínica de insuficiencia cardíaca congestiva o afectación pericárdica variable. Se localizan habitualmente en la aurícula derecha o en el septo interauricular, causando frecuentemente obstrucción de los vasos venosos de dichas cámaras ${ }^{(2)}$.
La enfermedad plasmocítica cardíaca conlleva un pronóstico reservado, con una supervivencia del $50 \%$ a los 15 meses desde el diagnóstico ${ }^{(3)}$.

Asimismo, presenta una respuesta variable e irregular a los regímenes quimioterápicos, que incluyen fármacos utilizados en el tratamiento de mieloma múltiple, como antraciclinas, melfalán, bortezomib, lenalidomida y corticosteroides, con resultados pobres en cuanto a la supervivencia libre de progresión ${ }^{(4)}$.

En su diagnóstico diferencial, por su preferencia por invadir la región del septo interauricular, se debe excluir la presencia de hipertrofia septal lipomatosa. Esta última se presenta ecocardiográficamente como un tabique interauricular de aspecto brillante, que de forma característica respeta la zona de la fosa oval. En la resonancia cardíaca muestra aspectos típicos de infiltración grasa como hiperintensidad en T1, pérdida de señal con supresión grasa y ausencia de realce tras administración de gadolinio(5).

El plasmocitoma cardíaco es una entidad muy rara, en la que son fundamentales el contexto clínico y la imagen cardíaca multimodal, especialmente la resonancia cardíaca y la PET, reservándose la realización de biopsia cardíaca para aquellos casos que presentan una etiología y un curso clínico inciertos.

\section{Conclusión}

La afectación plasmocítica cardíaca es altamente inusual y requiere un alto índice de sospecha en un contexto clínico compatible.

Para su diagnóstico es imprescindible la resonancia cardíaca y pruebas de medicina nuclear, así como la ecocardiografía para descartar compromiso hemodinámico.

En su diagnóstico diferencial debe incluirse especialmente la hiperplasia lipomatosa del septo, por su localización típica, siendo la resonancia cardíaca la prueba de imagen idónea para discernir ambas entidades.

\section{Ideas para recordar}

- El plasmocitoma cardíaco es una neoplasia rara que tiende a localizarse en la aurícula derecha y septo interauricular.

- Para su diagnóstico es fundamental un alto índice de sospecha, además de la imagen cardíaca multimodal, especialmente la resonancia cardíaca.

\section{Bibliografía}

1. Coakley M, Yeneneh B, Rosenthal A, et al. Extramedullary cardiac multiple myeloma-A case report and contemporary review of the literature. Clin Lymphoma Myeloma Leuk 2016; 16: 246-252.

2. Nam JH, Shim CY, Kim D, et al. A solitary cardiac plasmacytoma: features in multimodality imaging. Eur Heart J Cardiovasc Imaging 2017; 18 (8): 941.

3. Toocheck C, Pinkhas D. Treatment of relapsed multiple myeloma complicated by cardiac extramedullary plasmacytoma with D-PACE chemotherapy. BMJ Case Rep 2018; 2018: bcr2017223611.

4. Alexiou C, Kau RJ, Dietzfelbinger $\mathrm{H}$, et al. Extramedullary plasmacytoma: tumor occurrence and therapeutic concepts. Cancer 1999; 85: 2305-2314.

5. Maleszewski JJ, Bois MC, Bois PJ, et al. Neoplasia and the heart: pathological review of effects with clinical and radiological correlation. J Am Coll Cardiol 2018; 72 (2): 202-227. 